\title{
Photoionization of the valence orbitals of $\mathrm{OH}$
}

\author{
J. A. Stephens and V. McKoy \\ Arthur Amos Noyes Laboratory of Chemical Physics, a) California Institute of Technology, Pasadena, \\ California 91125
}

(Received 14 September 1987; accepted 13 October 1987)

\begin{abstract}
We report the results of studies of the photoionization cross sections and asymmetry parameters for the $3 \sigma$ and $1 \pi$ levels of $\mathrm{OH}$, corresponding to the production of the $A^{3} \Pi, c^{1} \Pi$, $a^{1} \Delta, b^{1} \Sigma^{+}$, and $X^{3} \Sigma^{-}$molecular ions. The calculations employed multiplet-specific HartreeFock potentials and numerical photoelectron continuum orbitals, obtained using the iterative Schwinger variational method. Noticeable nonstatistical behavior of the cross sections is seen, mainly for the $1 \pi$ level, although deviations are not as pronounced as in other open-shell systems. Comparison with fragmentary experimental data is encouraging, although synchrotron radiation studies are needed to fully assess the accuracy of the calculated cross sections.
\end{abstract}

\section{INTRODUCTION}

There has been only a limited amount of experimental investigation of the photoabsorption and photoionization processes of the $\mathrm{OH}$ radical. Van Lonkhuyzen and de Lange ${ }^{1}$ have recently employed novel techniques of ultraviolet photoelectron spectroscopy to accurately determine the ionization potentials leading to the ionic states $A^{3} \Pi, b^{1} \Sigma^{+}, a^{1} \Delta$, and $X^{3} \Sigma^{-}$. In addition to this fixed-wavelength study, Dehmer $^{2}$ has measured a high-resolution, relative photoionization cross section of $\mathrm{OH}$ from the $X^{3} \Sigma^{-}$ionic threshold to $\sim 3 \mathrm{eV}$ photoelectron kinetic energy, using the technique of photoionization mass spectrometry. Several members of a Rydberg series, superimposed on a background continuum, were observed and tentatively assigned as those converging to the $a^{1} \Delta$ ionic state. The only absolute determination of the photoexcitation of $\mathrm{OH}$ in the vacuum ultraviolet is the recent absorption cross section measurements by Nee and Lee. ${ }^{3}$ Several prominent discrete absorption peaks and an absorption continuum (corresponding to photodissociation) were observed. These observations compared favorably with extensive studies of the discrete excited states and calculations of the photodissociation cross section by van Dishoeck and Dalgarno. ${ }^{4}$

$\mathrm{OH}$ is an open-shell molecule with the ground state electronic configuration $1 \sigma^{2} 2 \sigma^{2} 3 \sigma^{2} 1 \pi^{3} X^{2} \Pi$. Ionization of a $1 \pi$ electron results in the $b^{1} \Sigma^{+}, a^{1} \Delta$, and the $X^{3} \Sigma^{-}$ionic terms, while ionization of the $3 \sigma$ electron leads to the $A^{3} \Pi$ and $c^{1} \Pi$ terms. The $c^{1} \Pi$ ionic state has not been observed experimentally, although it has been studied by accurate $a b$ initio calculations. ${ }^{5,6}$

In this paper, we present $a b$ initio calculations of photoionization cross sections and photoelectron angular distributions for the $3 \sigma$ and $1 \pi$ levels of $\mathrm{OH}$, corresponding to the formation of the first five accessible states of $\mathrm{OH}^{+}$. The photoionization dynamics of this important diatomic molecule does not appear to have been previously studied theoretically. Likewise, there are no measurements of absolute partial

\footnotetext{
a) Contribution No. 7662 .
}

photoionization cross sections or angular distributions available for direct comparison with the present calculations. We hope the present work will stimulate further theoretical and experimental investigation of the photoionization of $\mathrm{OH}$. Furthermore, these single-photon ionization studies are a first step in our planned studies of the resonant multiphoton ionization of $\mathrm{OH}$, an important radical in molecular photofragmentation.

\section{THEORY}

\section{A. Multiplet-specific wave functions and potentials}

In this section we give the explicit forms of the multiplet-specific continuum wave functions and the HartreeFock static-exchange potentials appropriate for ionization of the $3 \sigma$ and $1 \pi$ levels of $\mathrm{OH}$. There have been few theoretical studies of the photoionization of open-shell molecules which employ multiplet-specific ion potentials. Multipletspecific photoionization calculations have recently been reported for the $4 \sigma$ and $5 \sigma$ levels of NO. ${ }^{7,8}$ Significant nonstatistical behavior in photoionization cross sections for the ${ }^{1} \Pi$ and ${ }^{3} \Pi$ ionic states were noted, and attributed mainly to the $k \sigma$ shape resonance. ${ }^{9-12}$ Because multiplet-specific potentials differ in their exchange components, the short-range, electron-molecule interactions which are responsible for shape resonances should be somewhat sensitive to differences in the ion potentials. For NO, differences in the exchange interaction resulted in the shape resonance peaking at different photoelectron kinetic energies for the two ions $(\Delta \epsilon \sim 3 \mathrm{eV})$, and significant deviation from the 3:1 statistical ratio which results from a multiplet-averaged calculation. ${ }^{12,13}$ The use of a multiplet-averaged potential corresponds to the photoelectron wave function being identical for the several ionic states formed upon removal of a bound electron from an open-shell system, and cannot account for such effects.

For $\mathrm{OH}$, ejection of an electron from the $3 \sigma$ orbital produces the $A^{3} \Pi$ and $c^{1} \Pi$ ion states with ionization potentials of 16.48 and $19.21 \mathrm{eV}$, respectively. ${ }^{1,5}$ The final-state continuum wave functions for photoionization leading to the $A^{3} \Pi$ ion are 


$$
\begin{aligned}
& \Psi\left({ }^{2} \Pi\right)=1 / \sqrt{6}\left\{2 \mid[\text { core }] 3 \sigma 1 \pi_{+} \overline{k \sigma}|-|[\text { core }] \overline{3 \sigma} 1 \pi_{+} k \sigma|-|[\text { core }] 3 \sigma \overline{1}_{+} k \sigma \mid\right\}, \\
& \Psi\left({ }^{2} \Sigma^{+}\right)=1 / \sqrt{12}\left\{2\left|[\operatorname{core}] 3 \sigma 1 \pi_{+} \overline{k \pi}_{-}\right|-\mid[\text {core }] \overline{3 \sigma} 1 \pi_{+} k \pi_{-}|-|[\text {core }] 3 \sigma \overline{1 \pi}_{+} k \pi_{-} \mid\right. \\
& \left.+2\left|[\operatorname{core}] 3 \sigma 1 \pi_{-} \overline{k \pi}_{+}\right|-\left|[\operatorname{core}] \overline{3 \sigma} 1 \pi_{-} k \pi_{+}\right|-\left|[\operatorname{core}] 3 \sigma \overline{1 \pi} \bar{t}_{-} k \pi_{+}\right|\right\} \text {, } \\
& \Psi\left({ }^{2} \Sigma^{-}\right)=1 / \sqrt{12}\left\{2 \mid[\text { core }] 3 \sigma 1 \pi_{+} \overline{k \pi}_{-}|-|[\text {core }] \overline{3 \sigma} 1 \pi_{+} k \pi_{-}|-|\left[\text {core] } 3 \sigma \overline{1}_{+} k \pi_{-} \mid\right.\right. \\
& \left.-2 \mid[\text { core }] 3 \sigma 1 \pi_{-} \overline{k \pi}_{+}|+|[\text {core }] \overline{3 \sigma} 1 \pi_{-} k \pi_{+}|+|[\text {core }] 3 \sigma \overline{1 \pi}_{-} k \pi_{+} \mid\right\} \text {, } \\
& \Psi\left({ }^{2} \Delta\right)=1 / \sqrt{6}\left\{2\left|[\operatorname{core}] 3 \sigma 1 \pi_{+} \overline{k \pi}_{+}\right|-\mid[\text {core }] \overline{3 \sigma} 1 \pi_{+} k \pi_{+}|-|[\text {core }] 3 \sigma \overline{1} \pi_{+} k \pi_{+} \mid\right\},
\end{aligned}
$$

where [core] $=1 \sigma^{2} 2 \sigma^{2} 1 \pi^{2}$. The final-state continuum wave functions for photoionization leading to the $c^{1} \Pi$ ion are

$$
\begin{aligned}
\Psi\left({ }^{2} \Pi\right)= & 1 / \sqrt{2}\left\{\mid[\text { core }] 3 \sigma \overline{1 \pi}_{+} k \sigma \mid\right. \\
- & \left.\mid[\text { core }] \overline{3 \sigma} 1 \pi_{+} k \sigma \mid\right\}, \\
\Psi\left({ }^{2} \Sigma^{+}\right)= & \frac{1}{2}\left\{\mid[\text { core }] 3 \sigma \overline{1 \pi} \pi_{+} k \pi_{-} \mid\right. \\
& -\mid[\text {core }] \overline{3 \sigma} 1 \pi_{+} k \pi_{-} \mid \\
& +\mid[\text {core }] 3 \sigma \overline{1 \pi}+k \pi_{+} \mid \\
& \left.-\mid[\text {core }] \overline{3 \sigma} 1 \pi_{-} k \pi_{+} \mid\right\}, \\
\Psi\left({ }^{2} \Sigma^{-}\right)= & \frac{1}{2}\left\{\mid[\text { core }] 3 \sigma \overline{1 \pi} \pi_{+} k \pi_{-} \mid\right. \\
& -\mid[\text {core }] \overline{3 \sigma} 1 \pi_{+} k \pi_{-} \mid \\
& -\mid[\text {core }] 3 \sigma \overline{1 \pi_{-}} k \pi_{+} \mid \\
& \left.+\mid[\text {core }] \overline{3 \sigma} 1 \pi_{-} k \pi_{+} \mid\right\}, \\
\Psi\left({ }^{2} \Delta\right)=1 / \sqrt{2}\left\{\mid[\text { core }] 3 \sigma \overline{1 \pi}_{+} k \pi_{+} \mid\right. & \left.-\mid[\text {core }] \overline{3 \sigma} 1 \pi_{+} k \pi_{+} \mid\right\},
\end{aligned}
$$

with the same definition of the core electrons as above.

Removal of an electron from the $1 \pi$ orbital of $\mathrm{OH}$ produces the $X^{3} \Sigma^{-}, a^{1} \Delta$, and $b^{1} \Sigma^{+}$ionic states, with ionization potentials of $13.01,15.17$, and $16.61 \mathrm{eV}$, respectively. ${ }^{1}$ The final state continuum wave functions of photoionization of the $1 \pi$ orbital leading to the $X^{3} \Sigma^{-}$ion are

$$
\begin{aligned}
\Psi\left({ }^{2} \Pi\right)= & 1 / \sqrt{6}\left\{2 \mid[\text { core }] 1 \pi_{-} 1 \pi_{+} \overline{k \pi}_{+} \mid\right. \\
& -\mid[\text {core }] 1 \pi_{-} \overline{1 \pi}_{+} k \pi_{+} \mid \\
& \left.-\mid[\text {core }] \overline{1} \bar{\pi}_{-} 1 \pi_{+} k \pi_{+} \mid\right\}, \\
\Psi\left({ }^{2} \Sigma^{-}\right)= & 1 / \sqrt{6}\left\{2 \mid[\text { core }] 1 \pi_{-} 1 \pi_{+} \overline{k \sigma} \mid\right. \\
& -\mid[\text { core }] 1 \pi_{-} \overline{1}_{+} k \sigma \mid \\
& \left.-\mid[\text { core }] \overline{1 \pi_{-}} 1 \pi_{+} k \sigma \mid\right\}, \\
\Psi\left({ }^{2} \Delta\right)= & 1 / \sqrt{6}\left\{2 \mid[\text { core }] 1 \pi_{-} 1 \pi_{+} \overline{k \delta}+\mid\right. \\
& -\mid[\text { core }] 1 \pi_{-} \overline{1}_{+} k \delta_{+} \mid \\
& \left.-\mid[\text {core }] \overline{1 \pi}_{-} 1 \pi_{+} k \delta_{+} \mid\right\},
\end{aligned}
$$

where $[$ core $]=1 \sigma^{2} 2 \sigma^{2} 3 \sigma^{2}$. The final-state continuum wave functions for photoionization of the $1 \pi$ orbital leading to the $a^{1} \Delta$ ion are

$$
\begin{aligned}
& \Psi\left({ }^{2} \Pi\right)=\mid {[\text { core }] 1 \pi_{-} \overline{1}_{-} k \pi_{+} \mid } \\
& \Psi\left({ }^{2} \Sigma^{+}\right)= 1 / \sqrt{2}\left\{\mid[\text { core }] 1 \pi_{+} \overline{1 \pi}_{+} k \delta_{-} \mid\right. \\
&\left.+\mid[\text {core }] 1 \pi_{-} \overline{1 \pi}_{-} k \delta_{+} \mid\right\} \\
& \Psi\left({ }^{2} \Sigma^{-}\right)= 1 / \sqrt{2} \mid[\text { core }] 1 \pi_{+} \overline{1 \pi}_{+} k \delta_{-} \mid \\
&\left.-\mid[\text {core }] 1 \pi_{-} \overline{1 \pi}_{-} k \delta_{+} \mid\right\} \\
& \Psi\left({ }^{2} \Delta\right)=\mid[\text { core }] 1 \pi_{-} \overline{1 \pi}_{-} k \sigma \mid .
\end{aligned}
$$

Those for photoionization of the $1 \pi$ orbital leading to the $b^{1} \Sigma^{+}$ion are

$$
\begin{aligned}
\Psi\left({ }^{2} \Pi\right)= & 1 / \sqrt{2}\left\{\mid[\text { core }] 1 \pi_{-} \overline{1 \pi} \pi_{+} k \pi_{+} \mid\right. \\
& -\mid\left[\text {core] } \overline{1 \pi_{-}} 1 \pi_{+} k \pi_{+} \mid\right\}, \\
\Psi\left({ }^{2} \Sigma^{+}\right)= & 1 / \sqrt{2}\left\{\mid[\text { core }] 1 \pi_{-} \overline{1}_{+} k \sigma \mid\right. \\
& \left.+\mid[\text { core }] 1 \pi_{+} \overline{1 \pi}_{-} k \sigma \mid\right\}, \\
\Psi\left({ }^{2} \Delta\right)=1 / \sqrt{2}\left\{\mid[\text { core }] 1 \pi_{-} \overline{1 \pi}_{+} k \delta_{+} \mid\right. & \left.-\mid[\text {core }] \overline{1 \pi}-1 \pi_{+} k \delta_{+} \mid\right\} .
\end{aligned}
$$

With these wave functions the static-exchange, one-particle Schrödinger equations for the continuum orbitals $\phi_{\mathbf{k}}$ are derived ${ }^{14,15}$ by evaluation of the variational expression $\langle\delta \Psi|H-E| \Psi\rangle=0$, where $H$ is the fixed-nuclei Hamiltonian, and $E$ is the total energy. They are of the form

$$
\begin{array}{r}
P\left[f+\sum_{\text {core }}\left(2 J_{i}-K_{i}\right)+a_{n} J_{n}+b_{n} K_{n}\right. \\
\left.+\alpha S_{1 \pi}^{\prime \prime}+\beta S_{i_{\pi}}^{\prime}-\epsilon\right] P\left(\phi_{\mathbf{k}}\right\rangle=0
\end{array}
$$

where $J_{i}$ and $K_{i}$ are the Coulomb and exchange operators, and $P$ is a projection operator which enforces orthogonality of the continuum orbital to the occupied orbitals. ${ }^{14,15}$ The photoelectron kinetic energy is given by $\epsilon=\frac{1}{2} k^{2}$. The operators $S^{\prime \prime}$ and $S^{\prime}$ are defined by 


$$
S_{\pi}^{\prime \prime} \phi_{+}\left(\mathbf{r}_{1}\right)=\phi_{-}\left(\mathbf{r}_{1}\right) \int d^{3} r_{2}\left[\pi_{-}\left(\mathbf{r}_{2}\right)\right]^{*} \frac{1}{r_{12}} \pi_{+}\left(\mathbf{r}_{2}\right)
$$

and

$$
S_{n}^{\prime} \phi_{+}\left(\mathbf{r}_{1}\right)=\pi_{+}\left(\mathbf{r}_{1}\right) \int d^{3} r_{2}\left[\pi_{-}\left(\mathbf{r}_{2}\right)\right]^{*} \frac{1}{r_{12}} \phi_{-}\left(\mathbf{r}_{2}\right) .
$$

The one-electron operator in Eq. (6) is

$$
f=-\frac{1}{2} \nabla_{i}^{2}-\sum_{\alpha} \frac{Z_{\alpha}}{r_{i \alpha}},
$$

where $Z_{\alpha}$ is a nuclear charge. Using the wave functions given by Eqs. (1)-(5), the coefficients $a_{n}, b_{n}, \alpha$, and $\beta$ in Eq. (6) have been derived for the five ionic states of $\mathrm{OH}$ treated here, and are summarized in Table $I$.

\section{B. Calculational detalls}

The continuum solutions of the static-exchange equations were obtained using the iterative Schwinger variational method. The theory and numerical implementation of this technique have been fully discussed elsewhere. ${ }^{14,15}$ In the present calculations, the method of Smith et al. ${ }^{16}$ was used to solve the Lippmann-Schwinger equations associated with Eq. (6) for the continuum orbital $\phi_{\mathbf{k}}$. In this method the direct electrostatic potential in Eq. (6) is treated exactly by numerical integration, and the nonlocal, short-range exchange component is treated by expressing it in the approximate separable form:

$$
U_{\mathrm{ex}}\left(\mathbf{r}, \mathbf{r}^{\prime}\right)=\sum_{i, j}\left\langle\mathbf{r}\left|U_{\mathrm{ex}}\right| \alpha_{i}\right\rangle\left[U_{\mathrm{ex}}^{-1}\right]_{i, j}\left\langle\alpha_{j}\left|U_{\mathrm{ex}}\right| \mathbf{r}^{\prime}\right\rangle .
$$

Solutions obtained with this separable potential can be iteratively improved to yield the converged solutions of the Lippmann-Schwinger equations. ${ }^{16}$ This method is particularly

TABLE I. Coefficients of static-exchange potential in Eq. (6).

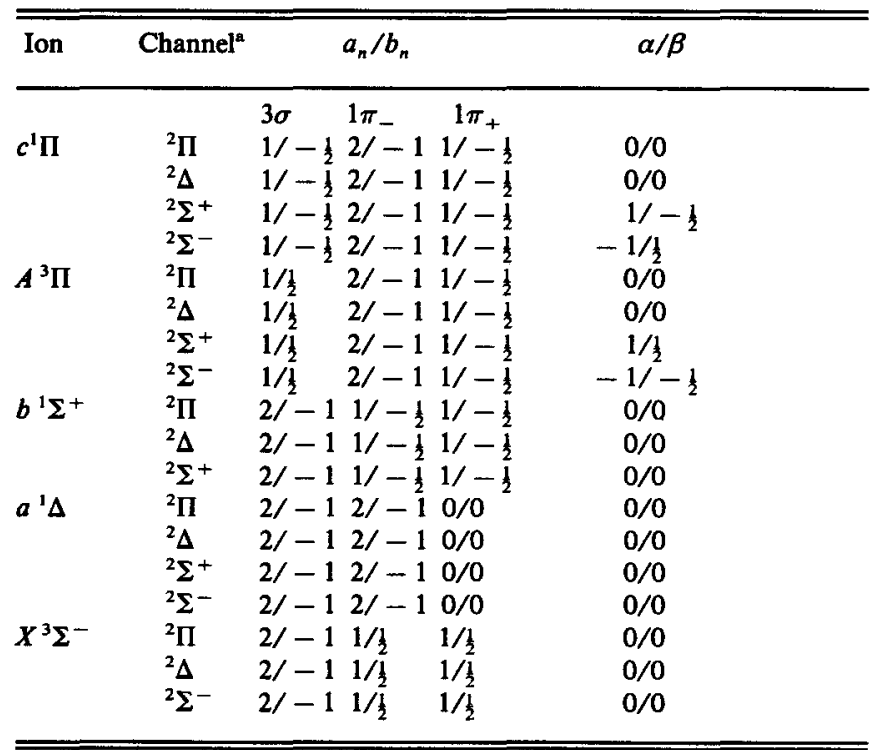

"Channel designates symmetry of the ion plus photoelectron system. suited to polar molecules, and with an appropriate choice of basis functions $\left\langle\mathbf{r} \mid \alpha_{i}\right\rangle$ (usually Cartesian Gaussian functions), solutions without iteration are generally well converged. This was found to be the case in the present application to $\mathrm{OH}$, as well as in NO. ${ }^{7}$

The initial state SCF wave function for $\mathrm{OH}$ was obtained with a [ $5 s 3 p 2 d / 3 s 2 p]$ basis of contracted Cartesian Gaussian functions. ${ }^{17-19}$ The oxygen $d$-function exponents are 1.2188 and 0.3611 , which are appropriate for representing a Slater-type orbital with $\xi_{s}=1.93$. The hydrogen $p$ function exponents are 2.5752 and 0.5991 (for $\xi_{s}=2.15$ ), and those for the $s$ functions 21.7208, 3.2729, 0.7377, and 0.2004 (for $\xi_{s}=1.275$ ) ${ }^{17,18}$ At the equilibrium internuclear distance of $R=1.832$ a.u., the total SCF energy in this basis was -75.4091 a.u.

In these calculations, all matrix elements and functions used in solution of the Lippmann-Schwinger equations corresponding to Eq. (6) were evaluated by employing singlecenter expansions about the center of mass. For converged cross sections and asymmetry parameters the following partial wave expansion parameters were established:

(i) maximum partial wave of the photoelectron continuum orbital $=8$;

(ii) maximum partial wave expansion of bound orbitals in the direct potential $=60$;

(iii) maximum partial wave expansion of $1 \sigma, 2 \sigma, 3 \sigma$, and $1 \pi$ bound orbitals in the exchange potential $=30$, 20,15 , and 15 , respectively;

(iv) maximum partial wave expansion of $1 / r_{12}$ in the direct and exchange terms $=60$ and 30 , respectively;

(v) maximum partial wave expansion of the nuclear potential $=60$.

The Gaussian basis sets used in the representation of the exchange potential Eq. (6) are listed in Table II, and were centered on the nuclei. The radial integration grid extended to 60 a.u. and contained 500 points. The integration step sizes ranged from 0.01 to 0.04 a.u. up to 5 a.u., and up to 0.4 a.u. beyond this point.

TABLE II. Basis sets used in separable potential, Eq. (10).

\begin{tabular}{cccc}
\hline \hline Symmetry & Center & $(l, m, n)^{\mathrm{a}}$ & Exponent \\
\hline$\sigma$ & $\mathrm{O}$ & $(0,0,0)$ & $8.0,4.0,2.0,1.0,0.5$ \\
& $\mathrm{O}$ & $(0,0,1)$ & $1.0,0.25$ \\
& $\mathrm{H}$ & $(0,0,0)$ & $1.2,0.4,0.1$ \\
& $\mathrm{H}$ & $(0,0,1)$ & $1.2,0.4,0.1$ \\
$\pi$ & $\mathrm{O}$ & $(1,0,0)$ & $8.0,4.0,2.0,1.0,0.5$ \\
& $\mathrm{O}$ & $(1,0,1)$ & $1.0,0.25$ \\
& $\mathrm{H}$ & $(1,0,0)$ & $1.2,0.4,0.1$ \\
& $\mathrm{H}$ & $(1,0,1)$ & $1.2,0.4,0.1$ \\
$\delta$ & $\mathrm{O}$ & $(1,1,0)$ & $8.0,4.0,2.0,1.0,0.5,0.25$ \\
& H & $(1,1,0)$ & $1.2,0.4,0.1$ \\
& & &
\end{tabular}

${ }^{2}$ Cartesian Gaussian basis functions defined as $\psi(r)=N\left(x-A_{x}\right)^{l}(y$ $\left.-A_{y}\right)^{m}\left(z-A_{z}\right)^{n} e^{-\alpha|\mathbf{r}-\mathbf{A}|^{2}}$. 


\section{RESULTS AND DISCUSSION}

\section{A. Photoionization of the $3 \sigma$ level}

In Fig. 1 we show our calculated $3 \sigma$ total cross sections for production of the $A^{3} \Pi$ and $c^{1} \Pi$ ions. The enhancement of the cross sections in Fig. 1 near the threshold results mainly from the kinetic energy dependence of the $k \sigma$ component. Examination of the eigenphase sum for this symmetry reveals that it is not shape resonant. This is not surprising, since the first-row hydrides are not expected to support molecular shape resonances. ${ }^{9}$ The $3 \sigma$ cross sections are quite similar, both in shape and magnitude, to ionization from the $1 b_{2}$ orbital of $\mathrm{H}_{2} \mathrm{O} .^{20,21}$ The bonding $3 \sigma$ and $1 b_{2}$ orbitals are both comprised mainly of the oxygen $2 p_{z}$ atomic orbital. On the other hand, photoionization from the nonbonding $1 \pi$ orbital leads to cross sections with substantially different spectral behavior, as discussed further below.

The ratio of the $A^{3} \Pi$ and $c^{1} \Pi$ cross sections are quite close to the statistical 3:1 ratio, with deviations typically $<7 \%$ over the kinetic energy range considered. As noted in Sec. II, much larger differences between multiplet-specific calculations for alternative ions have been predicted ${ }^{7,8}$ for the $4 \sigma$ and $5 \sigma$ levels of $\mathrm{NO}$, where the $k \sigma$ shape resonance is observed.

The calculated asymmetry parameter $\beta$ for the $c^{1} \Pi$ and $A^{3} \Pi$ ions are shown in Fig. 2. They are seen to be nearly equivalent beyond the $c^{1} \Pi$ threshold at $19.2 \mathrm{eV}$ photon energy, in accordance with the nearly statistical ratio predicted for the cross sections. There are no photoionization cross section or $\beta$ parameter measurements available for comparison with the present $3 \sigma$ results.

\section{B. Photoionization of the $1 \pi$ level}

Figure 3 shows our results for $1 \pi$ photoionization of $\mathrm{OH}$, corresponding to the production of the $X^{3} \Sigma^{-}, a^{1} \Delta$, and $b^{1} \Sigma^{+}$ionic states. The $1 \pi$ molecular orbital consists mainly of nonbonding oxygen $2 p_{x}$ and $2 p_{y}$ atomic orbitals,
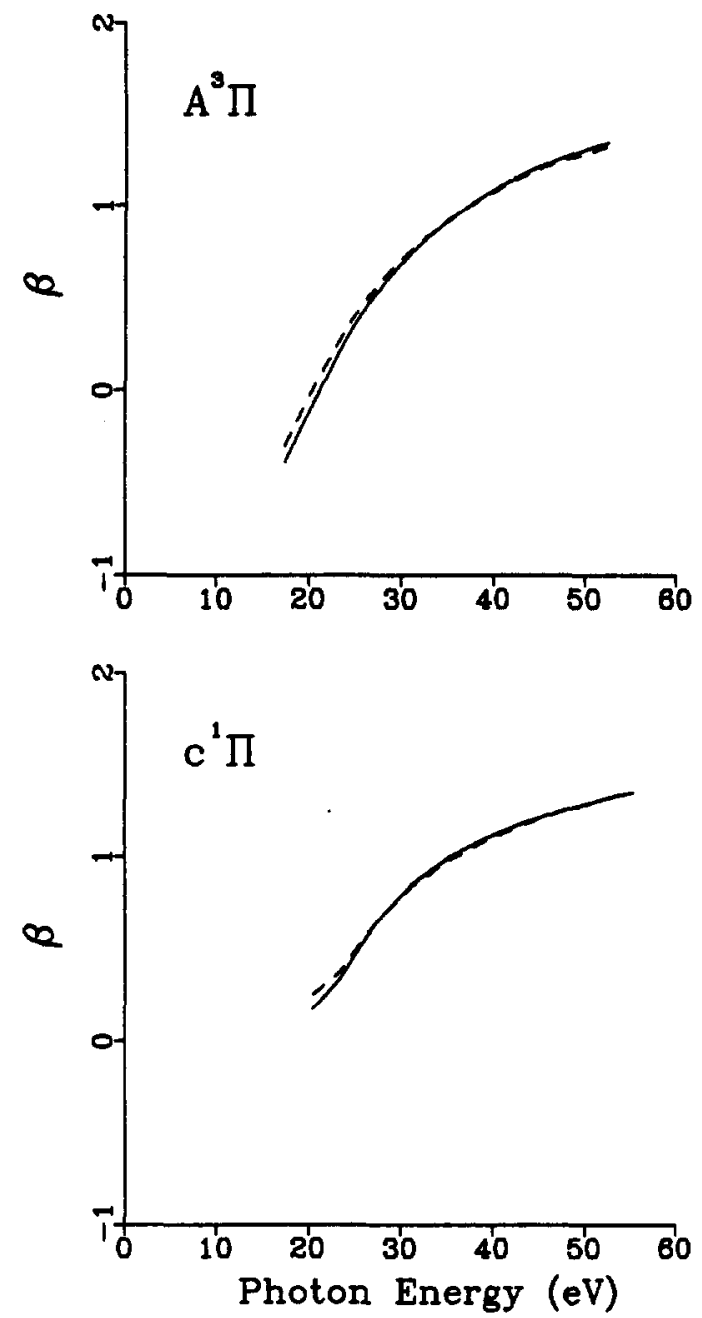

FIG. 2. Calculated asymmetry parameters for the $A^{3} \Pi$ and $c^{1} \Pi$ states of $\mathrm{OH}^{+}$(same designation as Fig. 1).

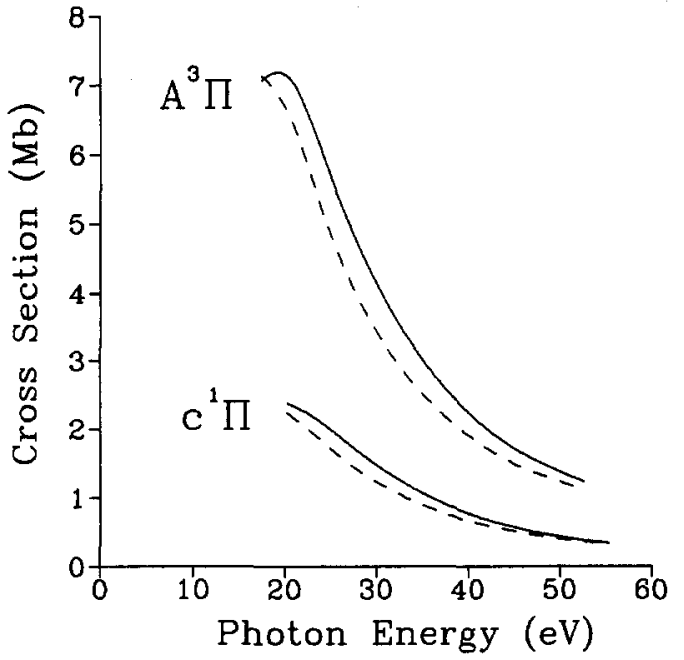

FIG. 1. Calculated photoionization cross sections for producing the $A^{3} \Pi$ and $c^{1} \Pi$ states of $\mathrm{OH}^{+} .(-)$results for dipole length form; (- - -) results for dipole velocity form.

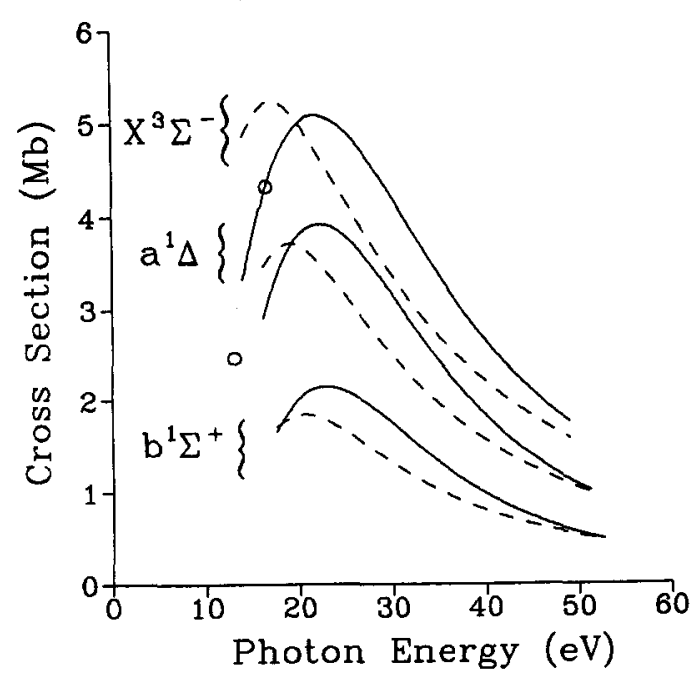

FIG. 3. Calculated photoionization cross sections for producing the $X^{3} \Sigma^{-}$, $a^{1} \Delta$, and $b^{1} \Sigma^{+}$states of $\mathrm{OH}^{+}$(same designation as in Fig. 1); $O$ experimental results from Ref. 2 (see explanation in the text). 
and the photoionization cross sections accordingly display different spectral variation compared to the $3 \sigma$ results. The shapes of the $1 \pi$ cross sections are quite similar to those obtained in multiplet-specific Hartree-Fock calculations on atomic oxygen. ${ }^{22,23}$ To emphasize this, in Fig. 4 we show the total $\mathrm{OH} 1 \pi$ level photoionization cross section, and compare it with the total atomic oxygen cross section, which has contributions from ${ }^{4} S,{ }^{2} D$, and ${ }^{2} P$ ionic terms. The enhancement in the $1 \pi$ cross section near $20-21 \mathrm{eV}$ is not due to a shape resonance, but results mainly from the energy dependence in the $1 \pi \rightarrow k \delta$ ionization channel. This simply parallels the dominant continuum $d$-wave contribution to $2 p$ photoionization of atomic oxygen, ${ }^{22,23}$ as well as other intense $n p \rightarrow \epsilon d$ continum transitions in the first-row atoms. Comparing our $\mathrm{OH} 1 \pi$ cross sections with those of water, ${ }^{20,21}$ we find fairly close resemblence to results for the nonbonding $1 b_{1}$ and $3 a_{1}$ orbitals. However, additional distortion of the oxygen atomic field by a second hydrogen causes the latter results to further deviate from the nearly atomic picture suggested in Fig. 4.

To a large extent, the probability of producing the $X^{3} \Sigma^{-}, a^{1} \Delta, b^{1} \Sigma^{+}$ions in the statistical ratio 3:2:1 is pre-
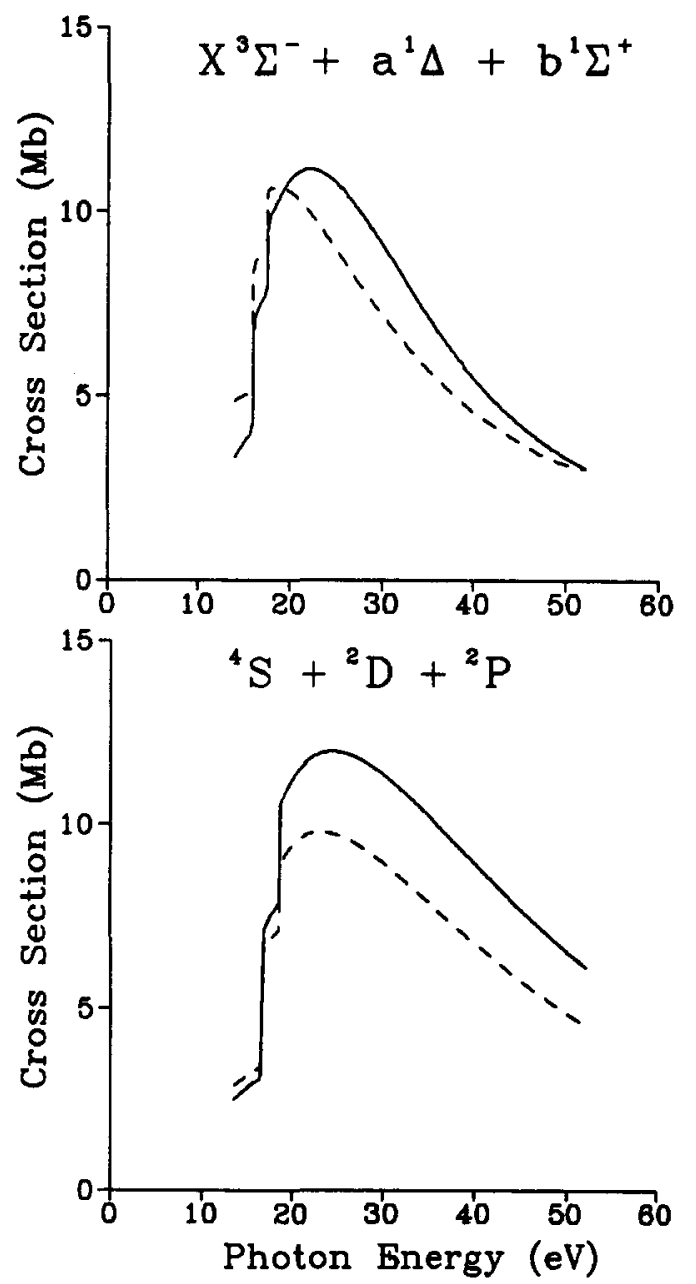

FIG. 4. Photoionization cross sections for $\mathrm{OH}$ and oxygen atom. Top: calculated total cross section for $1 \pi$ level of OH (same designation as Fig. 1). Bottom: calculated total cross section for $2 p$ photoionization of oxygen atom, from Ref. 23. dicted by the calculations over much of the kinetic energy range considered. The calculated cross sections deviate most significantly from this ratio in the region of the enhancement between 20 and $30 \mathrm{eV}$ photon energy, and mainly for the $X^{3} \Sigma^{-}$ion. At higher kinetic energies, effects due to the alternative exchange interactions for different ionic cores should be diminished. The calculated ratio of the $X^{3} \Sigma^{-}$, $a^{1} \Delta$, and $b^{1} \Sigma^{+}$cross sections are weakly energy dependent beyond $\sim 35 \mathrm{eV}$ photon energy, and at $\sim 50 \mathrm{eV}$ photon energy, the length and velocity form ratios are 3.0:1.9:1.0 and 2.9:2.0:1.0, respectively.

The calculated asymmetry parameters for the $1 \pi$ level are shown in Fig. 5. The $b^{1} \Sigma^{+}$and $a^{1} \Delta$ angular distributions are very similar, although the predicted differences beyond $\sim 25 \mathrm{eV}$ photon energy could be resolved by synchrotron measurements. The asymmetry parameter for the $X^{3} \Sigma^{-}$ion differs substantially from those for the other ions. These results, together with the deviations from statistical predictions for the $1 \pi$ cross sections at lower energy, indicate that open-shell effects may be more pronounced for pho-

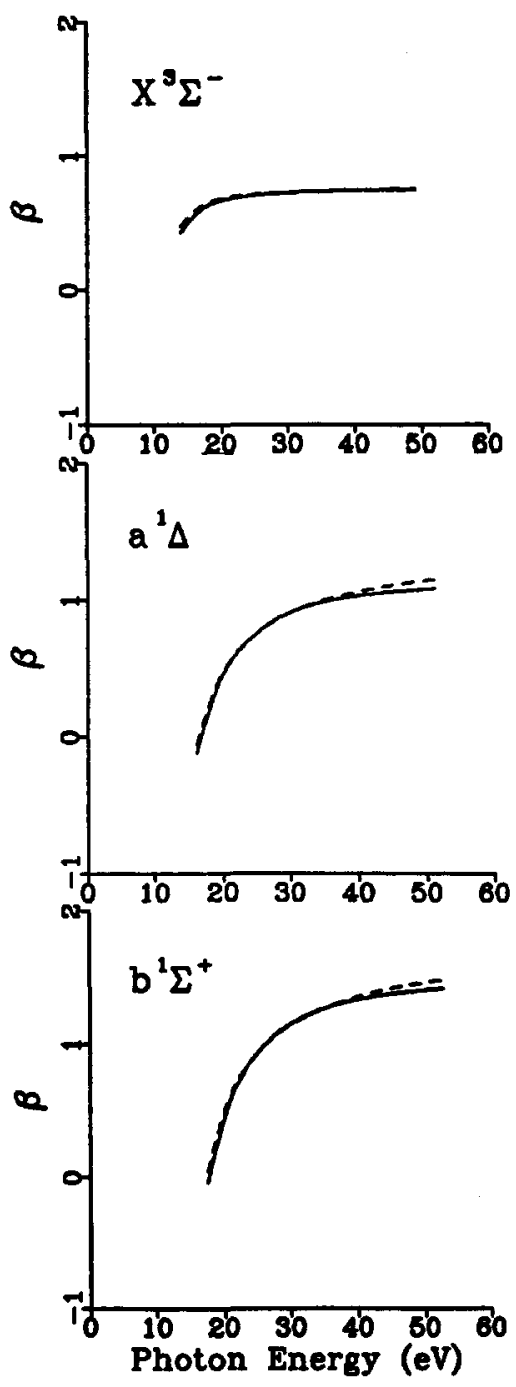

FIG. 5. Calculated asymmetry parameters for the $X^{3} \Sigma^{-}, a^{1} \Delta$, and $b^{1} \Sigma^{+}$ states of $\mathrm{OH}^{+}$(same designation as Fig. 1). 
toionization out of filled or partially filled degenerate molecular orbitals.

\section{Comparison with experiment}

For the $1 \pi$ level, there are two sources of experimental data which can be compared with the present calculations. First, van Lonkhuyzen and de Lange ${ }^{1}$ have measured the relative intensities of the $X^{3} \Sigma^{-}, a^{1} \Delta$, and $b^{1} \Sigma^{+}$ions to be $2.9 \pm 0.2,1.6 \pm 0.2$, and $1.0 \pm 0.2$, respectively, at $21.2 \mathrm{eV}$ photon energy ( $\mathrm{He}_{\alpha}$ line). If we convert these to branching ratios ${ }^{3} \Sigma^{-} /{ }^{1} \Sigma^{+}$and ${ }^{1} \Delta /{ }^{1} \Sigma^{+}$, and take the combined errors of the relative intensities into account, ${ }^{24}$ we find, respective$\mathrm{ly}$, the values $2.9 \pm 0.8$ and $1.6 \pm 0.5$. At this photon energy, our length form ${ }^{3} \Sigma-/{ }^{1} \Sigma^{+}$and ${ }^{1} \Delta /{ }^{1} \Sigma^{+}$branching ratios are 2.4 and 1.8 , respectively, in reasonable agreement with the above values. The corresponding velocity form branching ratios are 2.6 and 1.9 .

The relative photoionization cross section measured by Dehmer ${ }^{2}$ can be used to estimate the energy variation of the nonresonant, continuum background above the $X^{3} \Sigma^{-}$ threshold. In Fig. 3 we have included two data points, chosen at 13.1 and $16.5 \mathrm{eV}$ photon energy, which bracket the photon energy range employed by Dehmer. ${ }^{2}$ Normalizing the $16.5 \mathrm{eV}$ point to our calculated $X^{3} \Sigma^{-}$cross section (length form), the slope which extrapolates to threshold at $13.01 \mathrm{eV}$ is correctly reproduced. This conclusion remains valid if the data is normalized to the velocity form cross section.

\section{SUMMARY AND CONCLUSION}

In this paper we have studied the photoionization dynamics of the $3 \sigma$ and $1 \pi$ levels of $\mathrm{OH}$. Due to the open-shell structure of $\mathrm{OH}$, many more final-state channels are accessible than in closed-shell molecules. By employing multipletspecific potentials, the present calculations probe the different interactions between the photoelectron and alternative ionic cores. Since no shape resonances are present in $\mathrm{OH}$, the multiplet-specific calculations are generally in good accord with simple statistical predictions. However, for the $1 \pi$ level, predicted deviations of the cross sections from statistical behavior and differences between alternative angular distributions could likely be experimentally resolved.

Qualitatively, the present $\mathrm{OH}$ results compare with the oxygen atom and $\mathrm{H}_{2} \mathrm{O}$ results in a well-understood manner. The $1 \pi$ (nonbonding orbital) cross sections closely mirror the oxygen atom $2 p$ cross sections, and to a less extent the nonbonding $1 b_{1}$ and $3 a_{1}$ orbitals of water. Cross sections for the $3 \sigma$ (bonding orbital) more closely resemble those of the bonding $1 b_{2}$ orbital of water.

The branching ratios measured by van Lonkhuyzen and de Lange ${ }^{1}$ are in reasonable agreement with the present calculations, as well as the variation with photon energy of the (nonresonant) continuum background ${ }^{2}$ for producing the $X^{3} \Sigma^{-}$state a few electron volts above threshold. These comparisons are fragmentary, however, and experimental stud- ies employing tunable synchrotron radiation are needed to actually assess the accuracy of these calculations.

\section{ACKNOWLEDGMENTS}

This work was supported by grants from the National Science Foundation (CHE-8521391), Air Force Office of Scientific Research (Contract No. 87-0039), and the Office of Health and Environmental Research of the U.S. Department of Energy (DE-FG03-87ER60513). We also acknowledge use of resources of the San Diego SuperComputer Center, which is supported by the National Science Foundation. One of us (J.A.S.) wishes to thank Dr. Kimberly Schugart for helpful discussions and for pointing out Ref. 19.

'H. van Lonkhuyzen and C. A. de Lange, Mol. Phys. 51, 551 (1984),

${ }^{2}$ P. M. Dehmer, Chem. Phys. Lett. 110, 79 (1984).

${ }^{3}$ J. B. Nee and L. C. Lee, J. Chem. Phys. 81, 31 (1984).

${ }^{4}$ E. F. van Dishoeck and A. Dalgarno, J. Chem. Phys. 79, 873 (1983).

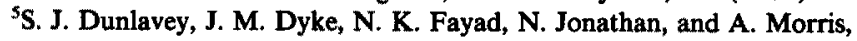
Mol. Phys. 38, 729 (1979).

${ }^{6}$ D. M. Hirst and M. F. Guest, Mol. Phys. 49, 1461 (1983).

'M. E. Smith, V. McKoy, and R. R. Lucchese, J. Chem. Phys. 82, 4147 (1985).

${ }^{8}$ M. R. Hermann, C. W. Bauschlicher, Jr., W. M. Huo, S. R. Langhoff, and P. W. Langhof, Chem. Phys. 109, 1 (1986).

'J. L. Dehmer, D. Dill, and A. C. Parr, in Photophysics and Photochemistry in the Vacuum Ultraviolet, edited by S. McGlynn, G. Findley, and R. Huebner (Reidel, Dordrecht, 1985), p. 341.

${ }^{10}$ J. L. Dehmer, A. C. Parr, and S. H. Southworth, in Handbook on Synchrotron Radiation, edited by G. V. Marr (North-Holland, Amsterdam, 1986), Vol. II.

${ }^{1} \mathrm{D}$. Lynch, V. McKoy, and R. R. Lucchese, in Symposium on Resonances in Electron Molecule Scattering, van der Waals Complexes, and Reactive Chemical Dynamics, edited by D. G. Truhlar, ACS Symp. Ser. 263 (American Chemical Society, Washington, D.C., 1984).

${ }^{12}$ S. Wallace, D. Dill, and J. L. Dehmer, J. Chem. Phys. 76, 1217 (1982).

${ }^{13}$ J. J. Delaney, I. H. Hillier, and V. R. Sanders, J. Phys. B 15, 1477 (1982).

${ }^{14}$ R. R. Lucchese, G. Raseev, and V. McKoy, Phys. Rev. A 25, 2572 (1982).

${ }^{15}$ R. R. Lucchese, K. Takatsuka, and V. McKoy, Phys. Rep. 131, 147 (1986), and references quoted therein.

${ }^{16}$ M. E. Smith, R. R. Lucchese, and V. McKoy, Phys. Rev. A 29, 1857 (1984)

${ }^{17}$ T. H. Dunning, J. Chem. Phys. 53, 2823 (1970).

${ }^{18}$ T. H. Dunning, J. Chem. Phys. 55, 3958 (1971).

${ }^{19}$ S. L. Guberman and W. A. Goddard III, J. Chem. Phys. 53, 1803 (1970). The $1 \pi_{x}$ and $1 \pi_{y}$ orbitals of the ground state SCF calculation used in the present calculation were symmetry averaged, as discussed in Sec. IV of this paper.

${ }^{20}$ G. H. F. Diercksen, W. P. Kraemer, T. N. Rescigno, C. F. Bender, B. V. McKoy, S. R. Langhoff, and P. W. Langhoff, J. Chem. Phys. 76, 1043 (1982).

${ }^{21} \mathrm{M}$. Braunstein and V. McKoy (to be published).

${ }^{22}$ A. Dalgarno, R. J. W. Henry, and A. L. Stewart, Planet. Space Sci. 12, 235 (1964).

${ }^{23}$ A. F. Starace, S. T. Manson, and D. J. Kennedy, Phys. Rev. A 9, 2453 (1974).

${ }^{24}$ When forming the branching ratios from the relative photoelectron intensities quoted in Ref. 1 , the relative errors should be summed to reflect the combined error in the ratio. 